\title{
A Trajectory Analysis of Alcohol and Marijuana Use Among Latino Adolescents in San Francisco, California
}

\author{
Sandra I. McCoy, Ph.D. ${ }^{a}{ }^{,}$, Nicholas P. Jewell, Ph.D. ${ }^{b}$, Alan Hubbard, Ph.D. ${ }^{b}$, Caitlin E. \\ Gerdts, M.H.S. ${ }^{\text {}}$, Irene A. Doherty, Ph.D. ${ }^{d}$, Nancy S. Padian, Ph.D. ${ }^{a}$, and Alexandra M. \\ Minnis, Ph.D. ${ }^{\mathrm{c}, \mathrm{e}}$ \\ anstitute of Business and Economic Research, University of California, Berkeley, California

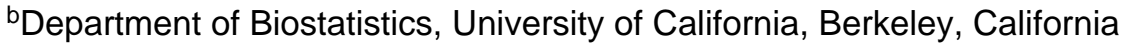 \\ 'Women's Global Health Imperative, RTI International, San Francisco, California \\ dSchool of Medicine, University of North Carolina, Chapel Hill, North Carolina \\ eDepartment of Epidemiology, University of California, Berkeley, California
}

\begin{abstract}
Purpose-We examined alcohol and marijuana use trajectories among Latino adolescents in the San Francisco Bay Area.

Methods-A total of 410 Latino adolescents aged 14-19 years were recruited from community venues from years 2001 to 2004 and followed up for 2 years. In separate models, we identified groups with similar temporal patterns of alcohol and marijuana use using semi-parametric latent group trajectory modeling. Multivariable multinomial logistic regression was used to identify factors associated with the probability of trajectory group membership.
\end{abstract}

Results-The use of alcohol (76\%) and marijuana (55\%) in the previous 6 months was common. Three alcohol-use trajectories were identified: low users (18\%), moderate users (37\%), and frequent users (45\%). Low alcohol users (vs. moderate users) were found to be younger in age, preferred Spanish language, and had more parental monitoring. Frequent users were more likely to be male, sexually active, gang exposed, and have less parental monitoring than moderate users. Similarly, three marijuana-use trajectories were identified: low users (36\%), moderate users (35\%), and frequent users (28\%), with similar correlates of group membership.

Conclusions-Urban Latino adolescents' substance use is shaped by complex cultural and environmental influences. Patterns of substance use emerge by early adolescence highlighting the need for timely intervention.

\section{Keywords}

Latinos; Adolescents; Substance use; Acculturation; Gangs; Marijuana; Alcohol

\footnotetext{
(C) 2010 Society for Adolescent Medicine. All rights reserved.

*Address correspondence to: Sandra I. McCoy, M.P.H., Ph.D., Institute of Business and Economic Research, University of California, Berkeley, F502 Haas Building, Berkeley, California. smccoy@berkeley.edu.
} 
Reducing substance use among adolescents in the United States constitutes a major public health priority. Although national rates of cigarette, alcohol, and marijuana use among high school students have decreased since the late 1990s [1-3], they remain alarmingly high, with $45 \%$ and $20 \%$ of students reporting alcohol and marijuana use in the last month, respectively $[1,4]$. Latino adolescents, the fastest growing minority group in the United States [5], report rates of alcohol and marijuana use similar to white teens in national school-based studies [4, 6], but they are more likely to have used cocaine, heroin, methamphetamine, and ecstasy as compared with both white and black students [4]. Because early substance use may be associated with a variety of adverse health and psychological outcomes, including future substance abuse and sexual risk-taking [7-10], understanding the patterns and predictors of adolescent substance use among Latinos is a prerequisite to the development of appropriate and effective interventions.

Latino adolescents must navigate a complex cultural environment which can affect their substance use choices. Acculturation, the multidirectional process by which immigrants and their families assimilate to the behaviors, beliefs, and values of a new culture while adapting those of their culture of origin, is a strong and consistent risk factor for substance use among Latino adolescents $[6,11,12]$. More acculturated youth are more likely to report use of alcohol and other substances than those less acculturated-thereby adopting the substance use behavior of non-Latino youth $[6,13,14]$. Furthermore, stress associated with the acculturation process can be experienced both by Latino immigrant adolescents as they cope with a new cultural environment, as well as by U.S.-born Latino adolescents as they negotiate a bicultural identity involving mainstream American values and those of their parent's culture $[11,13]$. Other research suggests that "acculturation gaps" between adolescents and their parents may be related to adolescent alcohol use through deterioration of the traditional Latino values of parental respect and familism, a cultural outlook emphasizing the importance of close family relationships [12, 13]. In addition to the cultural context, the social environment for youth may influence substance use choices, especially in urban areas where poverty, violence (often manifested as gang violence), crime, and substance use may be common [15].

Research on substance use by Latino adolescents has been conducted predominantly among school-based, nationally representative samples $[4,6,16]$, which may underestimate patterns of substance use. The high school dropout rate for Latinos is at least double the dropout rate of whites and blacks (22\% vs. $6 \%$ and $11 \%$, respectively) [17], and substance use is typically higher among out-of-school youth [18]. To overcome these limitations, we examined alcohol and marijuana use among in- and out-of-school Latino adolescents living in the San Francisco Bay Area who participated in the Mission Teen Health Project [19-22]. The study was designed to measure the risk behavior of a community sample of youth in a major metropolitan area. The goals of the analysis were to describe frequencies, patterns, and heterogeneity of alcohol and marijuana use to form contextually and culturally relevant interventions that could prevent substance use and abuse among urban Latino adolescents. 


\section{Materials and Methods}

\section{Study population}

The San Francisco Mission Teen Health Project was a 2-year prospective cohort study of 555 adolescents conducted from 2001 to 2004 . The purpose of the study was to describe social and sexual networks among adolescents in the community [19]. The Mission District is the vibrant cultural center of San Francisco's Latino population and home to nearly onethird of San Francisco's approximately 109,000 Latino residents [23]. However, household incomes are less than those of San Francisco overall, and the youth are regularly exposed to alcohol and drug use, prostitution, domestic and street violence, and gang activity [20, 22, 23]. This analysis is limited to 410 self-identified Latino participants.

\section{Study design}

The study has been described previously [19, 20, 22]. In brief, participants were recruited using three methods: venue-based recruitment throughout the Mission District, recruitment at community agencies, and friend referrals [22]. Eligible youth were aged 14-19 years, resided in the San Francisco Bay Area, and spoke English or Spanish. Participants were interviewed at baseline and reinterviewed every 6 months for 2 years (five visits). At each visit, a comprehensive social, reproductive health, and sexual network interview was conducted. Consent was obtained directly from adolescents aged 18 and 19 years. For adolescents aged 14-17 years, we obtained both parental consent and the participants' assent. The Committee for Human Research at the University of California, San Francisco, approved all study procedures and the Institutional Review Board at RTI International approved the secondary analysis.

\section{Main outcome measures}

Participants characterized alcohol and marijuana use at every visit ("About how often did you drink more than a few sips of beer, wine, a wine cooler, or hard liquor/use marijuana?") with a frequency scale ("a couple of times," "less than once a month," "not every week but at least once a month," "not every day, but at least once a week," and "every day"). These values were transformed to a scale of 0 to 5. Participants were also asked about getting drunk, parental and peer attitudes, and the sources for marijuana, its cost, and locations where it was being used.

\section{Covariates}

We examined several possible correlates of substance use. We assessed the role of school status (in- or out-of-school), sexual activity (vaginal or anal sex), participation in sports, and church attendance. Maternal education and living in crowded conditions (defined according to the U.S. Census [24]) served as proxies for socioeconomic status.

To assess the cultural status of participants, we measured country of birth, years in the United States, and immigrant generational status. First-generation participants were foreignborn; second-generation participants were born in the United States with foreign-born parents (one or both); and third-generation participants were born in the United States with U.S.-born parents. We assessed language use at home and also the language preference. 
We assessed parental monitoring with the help of four questions: "How much of the time do your parents/guardians: 1) generally know where you are; 2) expect you to call if you are going to be late; 3) know who you are spending time with; and 4) know what you are doing when you are away from home?" The average response using a 4-point scale (none to all of the time) was classified into having no adult caretaker, having high levels of parental monitoring (monitoring most to all of the time), or less than high levels of parental monitoring. Finally, we assessed gang exposure, including current membership or affiliation, and having a sexual partner in a gang.

\section{Statistical analysis}

We first examined baseline descriptive statistics of alcohol and marijuana use. To group subjects by common temporal patterns of substance use, we used a data-adaptive, groupbased trajectory modeling procedure. Group-based trajectory models identify distinctive groups of individuals with similar patterns of a behavior or outcome over time [25, 26]. The underlying model is a latent mixture polynomial model; the data-generating distribution consists of several unknown groups, each with a characteristic polynomial trend over time. Both the number of groups and the underlying degree of the polynomial are unknown, and thus model selection procedures are used to choose these parameter values. After the final model is determined, one can estimate the posterior probability of belonging to each group, which can also be made a function (through multinomial logistic regression) of baseline covariates $[25,27]$.

To determine the mixture model for both alcohol and marijuana use, we used the following methods. The optimal number of groups was determined by simultaneous considerations of fit (assessing change in the Bayesian Information Criterion [BIC] between models with successively more groups [27]), substantial knowledge about substance use patterns, and ease of interpretability. During this phase, all the group trajectories were set to a second order (quadratic) equation in time. After the appropriate number of groups was determined, individual group trajectories were adjusted to improve model fit by either adding or removing up to fourth order polynomial terms to the model or from the model, respectively, again choosing the model on the basis of change in BIC [25]. No covariates were added to the model at this time.

After the number of groups and model form were chosen, we then examined baseline factors associated with group membership using multinomial logistic regression [25]. The goal of the model was to predict an adolescent's future pattern of substance use, given their current age and baseline covariates, rather than making causal inferences of the associations. After one fixes the order of the polynomial and the number of groups, the group assignment model and trajectory model are estimated simultaneously as part of a joint likelihood; the consequence is that the standard errors for the coefficients in the multinomial logistic regression model implicitly account for uncertainties in both group membership and the model for the trajectories. However, the "parameters" relating to prediction of the group are not parameters in the formal sense, which would assume that the classes are fixed and not estimated from the data. In reality, the definition of a class is a complex random function of the observed data, and thus the inference provided must be considered informal. Because 
this analysis was exploratory in nature (and given the randomness of the definition of group), covariates with $p<.20$ in bivariable associations with trajectory groups by the Wald test were selected for inclusion in the final multivariable model, which also included age (years) at enrollment. We present odds ratios and 95\% confidence intervals, which represent the odds of group membership in a particular trajectory group as compared with a referent trajectory group. We selected one measure of acculturation (personal language preference) for inclusion in the multivariable models on the basis of strength and precision of the association with the group trajectories in bivariable models. Because the results were similar for bivariable and multivariable models, we only present the multivariable models. All analyses were performed using SAS 9.2 statistical software (SAS Institute, Inc, Cary, NC); trajectory analyses were conducted using a customized procedure (PROC TRAJ) available at http://www.andrew.cmu.edu/user/bjones/.

\section{Results}

Girls comprised 56\% of the 410 participants and the average age at enrollment was 16.4 years (Table 1). Most (65\%) of the participants were born in the United States. Spanish was spoken in $64 \%$ of the homes overall and exclusively spoken in $42 \%$ of homes. Current membership in or affiliation with a gang was reported by $14 \%$ of boys and $18 \%$ of girls. Leaving school before earning either a high school diploma or General Education Development test was reported by 43 (11\%) of the total participants. High levels of parental monitoring were reported by $45 \%$ of participants; $14 \%$ had no adult caregiver.

\section{Alcohol use}

Peers were perceived to be mostly indifferent (55\%) or approving (22\%) of alcohol use, whereas parents were perceived to be disapproving (92\%, Table 2). At enrollment, $76 \%$ of participants reported alcohol use in the previous 6 months and another $17 \%$ initiated alcohol use during the study follow-up period. Although boys and girls reported similar histories of alcohol use at baseline and initiation of use during the study, boys reported higher frequencies of use and of getting drunk. During the period of follow-up, $60 \%$ of boys reported drinking alcohol at least weekly in at least one 6-month interval as compared with $45 \%$ of girls $(p=.02)$, and $42 \%$ reported getting drunk at least weekly as compared with $24 \%$ of girls $(p<.01)$.

We selected a 3-group alcohol model with linear trajectories as satisfactory (Figure 1). Although the 5-group model provided the best statistical fit, the additional two groups in the 3- versus 5-group model described subtle variability in patterns of moderate use; therefore, the 3-group model was selected as the most interpretable and parsimonious. Given the number of groups, the linear model was chosen on the basis of change in BIC and statistical significance of higher order terms. A low-level group reported little use throughout followup (group 1: "low users"), representing $18 \%$ of the sample. A second group, group 2 ("moderate users"), represented $38 \%$ of the sample and was characterized by sporadic use at ages 14 and 15 years and then gradually increasing use over time, but this increase never went past the "less than once a month" level. Group 3 (i.e., "frequent users"), represented 
$45 \%$ of the sample, reported less than monthly use at the age of 14 years, but reported almost weekly use by their early 20 s.

We examined baseline characteristics associated with group membership with group 2 ("moderate users") as the referent group (Table 3). Characteristics associated with membership in the low use group as compared with the moderate use group included young age, Spanish as the language of preference, and having high levels of parental monitoring. Factors associated with membership in the frequent use group included being male, being sexually active at baseline, being exposed to a gang (membership, affiliation, or having a sexual partner in a gang), and having no adult caretaker or low levels of parental monitoring.

\section{Marijuana use}

Peers were perceived to be indifferent (50\%) or approving (28\%) of marijuana use, whereas parents were perceived to be disapproving (93\%) of use (Table 2). Marijuana use in the previous 6 months at baseline was common (55\%) and another $21 \%$ of participants first used or reinitiated marijuana use during the follow-up period. Sources of marijuana included friends ( $82 \%$ ), regular suppliers (53\% for boys and $45 \%$ for girls), boy- or girlfriends (21\% for boys and $37 \%$ for girls), or relatives (22\% of boys and 33\% of girls). Among those reporting baseline use, the highest average amount spent at any one time on marijuana was $\$ 12$ for girls and $\$ 36$ for boys. Marijuana was used commonly on the street or in parking lots $(78 \%)$, at friend's homes (75\%), or in a car (71\%). During the follow-up period, $31 \%$ of participants reported daily marijuana use during at least one 6-month interval.

We selected a linear model with three marijuana groups as best representing the data (Figure 1) on the basis of change in BIC. Group 1 ("low users") reported no marijuana use during the study and represented $36 \%$ of the sample. Group 2 ("moderate users," $35 \%$ ) consistently reported less than monthly marijuana use during follow-up, and group 3 ("frequent users," $28 \%$ ) reported high and increasing usage, starting at monthly use and subsequently reaching weekly use by the age of 20 years. Characteristics associated with membership in the low use group included preferring using either Spanish or Spanish and English equally, not being sexually active, not being exposed to a gang, having high levels of parental monitoring, and perceiving disapproving peer attitudes toward marijuana (Table 4). In contrast, characteristics associated with membership in the high frequency marijuana group included English being the language of preference, being sexually active, gang exposure, having either lower levels of parental monitoring or no adult caregiver, and perceiving permissive peer attitudes toward marijuana.

\section{Joint trajectories of alcohol and marijuana use}

Of the total participants, $28 \%$ were in both the alcohol and marijuana high use groups. The conditional probability of being in the high marijuana use group given membership in the high alcohol use group was $60 \%$. Conversely, the probability of being in the high alcohol use group conditional on membership in the high marijuana use group was 94\%. Few (16\%) participants were in both the alcohol and marijuana low use groups. 


\section{Discussion}

In this trajectory analysis of substance use among urban Latino adolescents, alcohol and marijuana use was common and increased steadily throughout adolescence and early adulthood. Patterns of usage were detectable early in adolescence: by the age of 14 and 15, groups of adolescents with the highest frequencies of alcohol and marijuana use were easily discernable and they remained the highest users during the course of the study. The public health implications are clear: substance use education and prevention programs must be implemented well before junior high and high school. The window of opportunity for prevention of high-frequency use seems to close by age 16, when many youth have already become regular users.

Although most research suggests that adolescent alcohol use is more common as compared with marijuana [11, 28, 29]; 77\% of the participants reported marijuana use during the study period, suggesting that marijuana is highly normalized in this population. Given that youth were recruited in public venues, it is challenging to compare our findings with school-based studies and to appropriately put these data in context. Hispanic participants in the Youth Risk Behavioral Survey reported a decrease in 30-day marijuana use since 1997 (from 29\% to 19\%) [30]; however, data from the 2008 Monitoring the Future project indicated that marijuana use in the last 12 months may have stabilized or increased after a long period of decline (32\% for 12 th graders) [3]. Our community-based sample is clearly a much higher risk group of youth. The prevalence of daily marijuana use in at least one 6-month interval in the study (31\%) is striking, underscoring the need for intensified public health efforts to prevent marijuana use.

An innovative feature of this analysis was the use of semi-parametric latent group trajectory modeling, a tool which identifies clusters of individuals with similar trajectories of a behavioral pattern over time [25]. Trajectories can be adjusted to improve fit, and timestable and time-varying co-variates can be added to the model. Graphical displays of data are easy to interpret and succinctly summarize large quantities of data. However, an important shortcoming is the assumption that the differences in trajectories of subjects within a group due only to independent and identically distributed random normal errors around a mean trajectory. One such reason for not allowing more error structure (e.g., random effects models) is that it can be hard to identify the maximum likelihood estimator and the resulting estimated quantities regarding group membership cannot be considered true fixed parameters anyway; therefore, the model assumptions are strong and no matter what augmentations are added to the model, the inference will be informal.

Spanish language preference (or equal preference for English), our proxy measure for acculturation, was associated with lesser use of both alcohol and marijuana use, consistent with previous reports [12-14]. This finding demonstrates the importance of "selective acculturation" whereby youth assimilate to the U.S. culture while retaining protective elements of their familial heritage [6]. Similarly, cultural values of familism and parental respect may be evident in higher levels of parental monitoring; another factor strongly associated with substance use trajectories in this study and others [31-33]. Thus, 
interventions designed to strengthen relationships between immigrant parents and their adolescent children may help to reduce substance abuse among Latino youth [34].

There appears to be a complex interplay of sexual activity, gang affiliation, and substance use in our sample. Baseline sexual activity and gang exposure were both associated with higher frequencies of alcohol and marijuana use. This relationship between sexual activity and substance use has been noted previously [7, 35, 36], suggesting that introduction to, use of, and/or the availability of alcohol and marijuana may be closely tied to having a sexual partner. The association between gang affiliation and substance use is not unexpected [37], yet is concerning given that $25 \%$ of participants had some form of gang exposure (directly or through a sexual partner). Furthermore, in the parent study, 27\% of sexually active female participants became pregnant during the follow-up period, and pregnancy was strongly related to male partner gang membership [20]. The implication is that juvenile gangs, substance use, and sexual risk-taking comprise a dangerous synergy, and that substance use prevention should be integrated with reproductive health efforts and youth gang prevention.

This study has several limitations. First, trajectory analysis identifies clusters of individuals with similar temporal behavioral patterns; however, individual patterns may differ from the group trajectories and/or may exhibit patterns from multiple group trajectories. Furthermore, high levels of missing data from the lowest and highest age ranges limit the reliability of estimates in these ranges. An optimal dataset to evaluate substance use would enroll and follow-up participants of the same age; we have attempted to control some of the confounding induced by differential selection in the cohort by adjusting for age in the multivariable models. Second, self-reported drug use may be subject to social desirability bias which could result in under- or overreporting of substance use. Third, it is possible that missing data are non-ignorable such that participants with the highest levels of substance use were more likely to miss follow-up interviews. Our baseline data suggested that this was not the case because levels of baseline alcohol and marijuana use were similar between participants who were lost to follow-up after the first visit $(n=27)$ and those who attended at least one follow-up visit. However, we do not know the subsequent substance use patterns of participants who did not return for interviews so this remains an important limitation of the study. In addition, the street-based recruiting strategy resulted in a higher proportion of foreign-born males than females because of the inclusion of sites where men might congregate to find work as day laborers. Furthermore, we were unable to analyze the results separately by country of origin and have used the term "Latino" to describe a heterogeneous group of adolescents. Finally, we used proxy measures of acculturation such as language preference and generational status instead of the degree to which adolescents identified with the values and attitudes of the host culture and their culture of origin [11]. Despite these shortcomings, this analysis has improved our understanding of substance use among the population of urban Latino youth in San Francisco; findings which are probably generalizable to other urban Latino youth.

Although national trends of adolescent substance use are encouraging [1-3], our study has illuminated a complex picture of substance use among a community sample of Latino youth both in and out of school. Patterns of use were established early in adolescence, suggesting a need for early intervention. The retention of cultural values seemed to be protective against 
substance use; however, the conflicting risks of youth gangs and sexual activity are also strong influences on adolescents' health behavior. Latino youth comprise a large and growing proportion of U.S. youth, and continued attention to reducing alcohol and marijuana use in urban Latino adolescent populations is clearly warranted.

\section{Acknowledgments}

The authors are grateful to the Mission Teen Health Project study coordinators Carla Rodas and Evan van Dommelen-Gonzalez. We would also like to thank the study staff, data managers, and the community agencies that supported our research activities, including Mission Neighborhood Centers, Boys and Girls Club of San Francisco: Columbia Park Unit, Jamestown Community Center, and the Real Alternatives Program. We would like to acknowledge the contributions of the Department of Obstetrics, Gynecology and Reproductive Sciences at the University of California, San Francisco in managing the awards that funded this research and in supporting study implementation and administration.

Financial support was provided by the National Institute of Allergy and Infectious Diseases, National Institutes of Health (R01-AI48749); National Institute of Child Health and Human Development, National Institutes of Health (K01 HD047434); University-wide AIDS Research Program, University of California (M00-SF-056 \& 057A).

\section{References}

1. Centers for Disease Control and Prevention. Trends in the Prevalence of Selected Risk Behaviors for All Students National YRBS: 1991-2007. Atlanta, GA: Centers for Disease Control and Prevention; 2008.

2. Johnston, LD.; O’Malley, PM.; Bachman, JG., et al. More good news on teen smoking: Rates at or near record lows. Ann Arbor, MI: University of Michigan News Service; 2008. Available at: http:// www.monitoringthefuture.org. Retrieved June 10, 2009.

3. Johnston, LD.; O'Malley, PM.; Bachman, JG., et al. Various stimulant drugs show continuing gradual declines among teens in 2008, most illicit drugs hold steady. Ann Arbor, MI: University of Michigan News Service; 2008. Available at: http://www.monitoringthefuture.org. Retrieved June $10,2009$.

4. Eaton DK, Kann L, Kinchen S, et al. Youth risk behavior surveillance-United States, 2007. MMWR Surveill Summ. 2008; 57:1-131. [PubMed: 18528314]

5. U.S. Census Bureau. Press Release: U.S. Hispanic Population Surpasses 45 Million: Now 15 Percent of Total. Washington, DC: U.S. Department of Commerce; 2008.

6. Wahl AM, Eitle TM. Gender, acculturation and alcohol use among Latina/o adolescents: A multiethnic comparison. J Immigr Minor Health. 2008; 12:153-165. [PubMed: 18807187]

7. Stueve A, O'Donnell LN. Early alcohol initiation and subsequent sexual and alcohol risk behaviors among urban youths. Am J Public Health. 2005; 95:887-893. [PubMed: 15855470]

8. Fergusson DM, Boden JM. Cannabis use and later life outcomes. Addiction. 2008; 103:969-76. discussion 977-8. [PubMed: 18482420]

9. Hayatbakhsh MR, Najman JM, Jamrozik K, et al. Cannabis and anxiety and depression in young adults: A large prospective study. J Am Acad Child Adolesc Psychiatry. 2007; 46:408-417. [PubMed: 17314727]

10. Larm P, Hodgins S, Larsson A, et al. Long-term outcomes of adolescents treated for substance misuse. Drug Alcohol Depend. 2008; 96:79-89. [PubMed: 18375076]

11. De La Rosa M. Acculturation and Latino adolescents' substance use: A research agenda for the future. Subst Use Misuse. 2002; 37:429-456. [PubMed: 12064428]

12. Martinez CR. Effects of differential family acculturation on Latino adolescent substance use. Fam Relat. 2006; 55:306-317.

13. Gil AG, Wagner EF. Acculturation, familism, and alcohol use among Latino adolescent males: Longitudinal relations. J Community Psychol. 2000; 28:443-458.

14. Epstein JA, Dusenbury L, Botvin GJ, et al. Language use and initiation of alcohol use among New York City Hispanic adolescents. J Child Adoles Subst Abuse. 1996; 5:63-79. 
15. Lambert SF, Brown TL, Phillips CM, et al. The relationship between perceptions of neighborhood characteristics and substance use among urban African American adolescents. Am J Community Psychol. 2004; 34:205-218. [PubMed: 15663207]

16. Delva J, Wallace JM Jr, O'Malley PM, et al. The epidemiology of alcohol, marijuana, and cocaine use among Mexican American, Puerto Rican, Cuban American, and other Latin American eighthgrade students in the United States: 1991-2002. Am J Public Health. 2005; 95:696-6702. [PubMed: 15798132]

17. Chavez EL, Edwards R, Oetting ER. Mexican American and white American school dropouts' drug use, health status, and involvement in violence. Public Health Rep. 1989; 104:594-604. [PubMed: 2511592]

18. Swaim RC, Beauvais F, Chavez EL, et al. The effect of school dropout rates on estimates of adolescent substance use among three racial/ethnic groups. Am J Public Health. 1997; 87:51-55. [PubMed: 9065226]

19. Doherty IA, Minnis A, Auerswald CL, et al. Concurrent partnerships among adolescents in a Latino community: The Mission District of San Francisco, California. Sex Transm Dis. 2007; 34:437-443. [PubMed: 17195772]

20. Minnis AM, Moore JG, Doherty IA, et al. Gang exposure and pregnancy incidence among female adolescents in San Francisco: Evidence for the need to integrate reproductive health with violence prevention efforts. Am J Epidemiol. 2008; 167:1102-1109. [PubMed: 18308693]

21. Auerswald CL, Greene K, Minnis A, et al. Qualitative assessment of venues for purposive sampling of hard-to-reach youth: An illustration in a Latino community. Sex Transm Dis. 2004; 31:133-138. [PubMed: 14743078]

22. Minnis, A.; Auerswald, C.; Doherty, I., et al. Qualitative and quantitative methods for developing a venue-based sampling approach for a study of Latino adolescents' social and sexual networks. Bethesda, MD: National Institute on Drug Abuse, National Institutes of Health; 2002.

23. Eiseman, M. Socio-Economic Profile of the Mission District. San Francisco, CA: Mission Economic Development Agency; 2006.

24. Bennefield, R.; Bonnette, R. Structural and Occupancy Characteristics of Housing, 2000. Washington, DC: U.S. Census Bureau, U.S. Department of Commerce; 2003.

25. Jones BL, Nagin DS. Advances in group-based trajectory modeling and an SAS procedure for estimating them. Sociol Methods Res. 2007; 35:542-571.

26. Nagin DS. Analyzing developmental trajectories: A semiparametric, group-based approach. Psychol Methods. 1999; 4:139-157.

27. Jones BL, Nagin DS, Roeder K. A SAS procedure based on mixture models for estimating developmental trajectories. Sociol Methods Res. 2001; 29:374-393.

28. Pantin HS, Sullivan S, Coatsworth J, et al. Preventing substance abuse in Hispanic immigrant adolescents: An ecodevelopmental, paren-centered approach. Hisp J Behav Sci. 2003; 25:469-500.

29. Strait S. Drug use among Hispanic youth: Examining common and unique contributing factors. Hisp J Behav Sci. 1999; 21:89-103.

30. Centers for Disease Control and Prevention. Trends in the Prevalence of Selected Risk Behaviors for Hispanic Students National YRBS: 1991-2007. Atlanta, GA: Centers for Disease Control and Prevention; 2008.

31. Allen ML, Elliott MN, Fuligni AJ, et al. The relationship between Spanish language use and substance use behaviors among Latino youth: A social network approach. J Adolesc Health. 2008; 43:372-379. [PubMed: 18809135]

32. Fulkerson JA, Pasch KE, Perry CL, et al. Relationships between alcohol-related informal social control, parental monitoring and adolescent problem behaviors among racially diverse urban youth. J Community Health. 2008; 33:425-433. [PubMed: 18607698]

33. Steinberg L, Fletcher A, Darling N. Parental monitoring and peer influences on adolescent substance use. Pediatrics. 1994; 93(6 Pt. 2):1060-1064. [PubMed: 8197008]

34. Prado G, Pantin H, Briones E, et al. A randomized controlled trial of a parent-centered intervention in preventing substance use and HIV risk behaviors in Hispanic adolescents. J Consult Clin Psychol. 2007; 75:914-926. [PubMed: 18085908] 
35. Anderson JE, Mueller TE. Trends in sexual risk behavior and unprotected sex among high school students, 1991-2005: The role of substance use. J Sch Health. 2008; 78:575-580. [PubMed: 18844809]

36. Guo J, Chung IJ, Hill KG, et al. Developmental relationships between adolescent substance use and risky sexual behavior in young adulthood. J Adolesc Health. 2002; 31:354-362. [PubMed: 12359381]

37. Thornberry TP, Krohn MD, Lizotte AJ, et al. The role of juvenile gangs in facilitating delinquent behavior. J Res Crime Delinq. 1993; 30:55-87. 


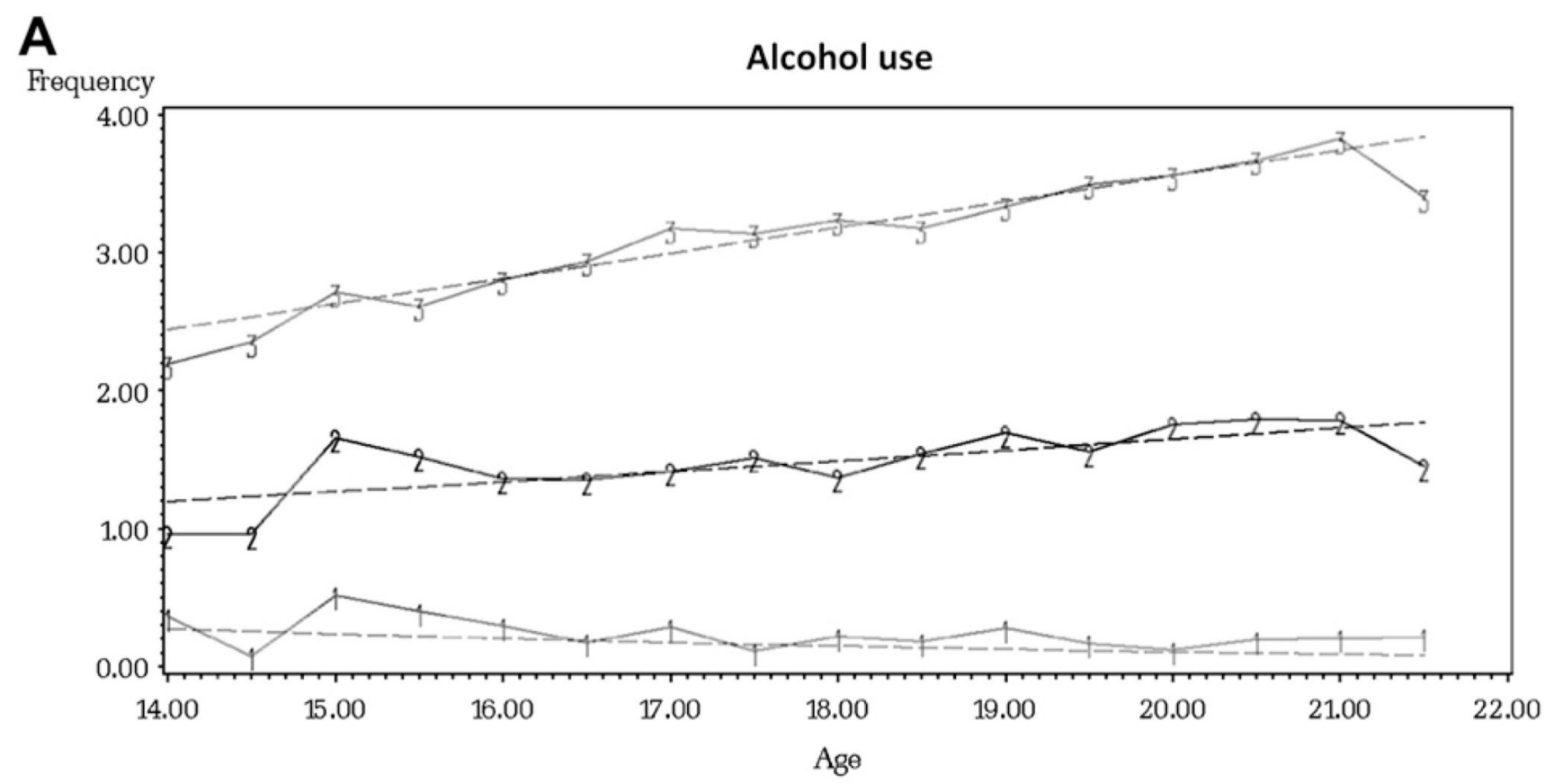

B

Frequency Marijuana use

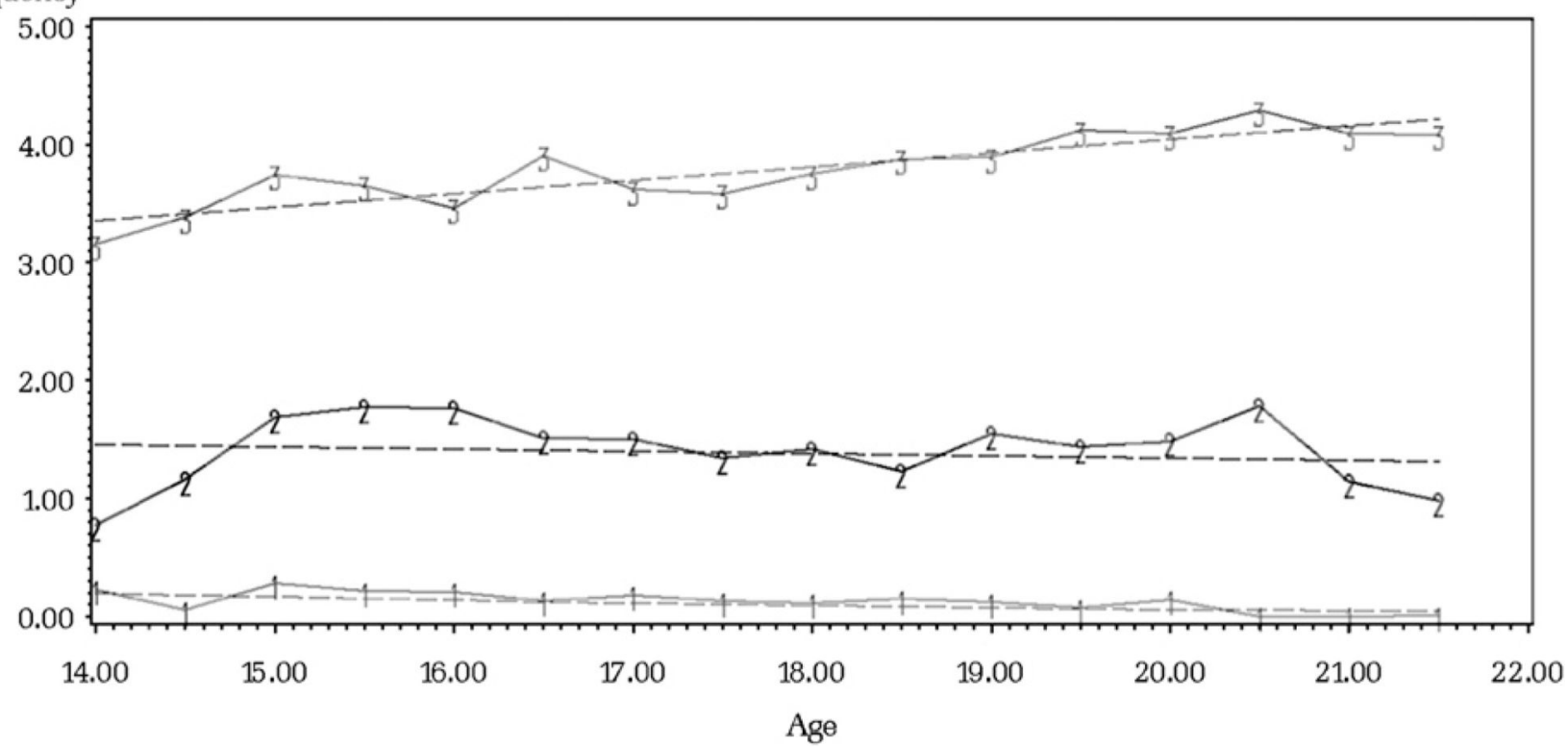

Figure 1.

Trajectories of alcohol (panel A) and marijuana use (panel B) among a sample of Latino youth in San Francisco, California. Age-specific observed (-) and predicted (---) substance use frequencies in the previous 6 months were reported at each of the five study visits with the use of the following scale: $0=$ no use, $1=$ a couple of times, $2=$ less than once a month, $3=$ not every week but at least once a month, $4=$ not every day but at least once a week, and 
$5=$ every day. Groups were identified by a given number $(1=$ low frequency, $2=$ moderate frequency, $3=$ high frequency). Trajectories are from the final model without covariates. 


\section{Table 1}

Baseline characteristics of 410 Latino youth participating in the Mission Teen Health Project, San Francisco, California, 2001-2004

\begin{tabular}{|c|c|c|c|}
\hline \multirow[t]{2}{*}{ Characteristic } & \multirow{2}{*}{$\begin{array}{c}\text { Overall }(\mathrm{n}=410)^{a} \\
\mathrm{~N}(\%)\end{array}$} & \multirow{2}{*}{$\frac{\text { Males }(n=180)}{N(\%)}$} & \multirow{2}{*}{$\frac{\text { Females }(\mathbf{n}=230)}{N(\%)}$} \\
\hline & & & \\
\hline \multicolumn{4}{|l|}{$\operatorname{Age}^{b}$} \\
\hline $14-15$ & $134(32.8)$ & $46(25.6)$ & $88(38.4)$ \\
\hline $16-17$ & $148(36.2)$ & $59(32.8)$ & 89 (38.9) \\
\hline $18-20$ & $127(31.1)$ & $75(41.7)$ & $52(22.7)$ \\
\hline \multicolumn{4}{|l|}{ Country of birth } \\
\hline United States & $267(65.1)$ & $103(57.2)$ & $164(71.3)$ \\
\hline Mexico & $78(19.0)$ & $42(23.3)$ & $36(15.7)$ \\
\hline El Salvador & $23(5.6)$ & $9(5.0)$ & $14(6.1)$ \\
\hline Guatemala & $14(3.4)$ & $10(5.6)$ & $4(1.7)$ \\
\hline Other & $28(6.8)$ & $16(8.9)$ & $12(5.2)$ \\
\hline \multicolumn{4}{|l|}{ Years in the United States } \\
\hline Less than 1 year & $15(3.7)$ & $9(5.0)$ & $6(2.6)$ \\
\hline At least 1 year & $126(30.9)$ & $67(37.4)$ & $59(25.8)$ \\
\hline Entire life (born in U.S.) & $267(65.4)$ & $103(57.5)$ & $164(71.6)$ \\
\hline \multicolumn{4}{|l|}{ Generational status } \\
\hline 1st generation & $143(34.9)$ & $77(42.8)$ & $66(28.7)$ \\
\hline 2nd generation & $211(51.5)$ & $79(43.9)$ & $132(57.4)$ \\
\hline 3rd generation or higher & $56(13.7)$ & $24(13.3)$ & $32(13.9)$ \\
\hline \multicolumn{4}{|l|}{ Language use at home } \\
\hline English & $146(36.0)$ & $53(29.9)$ & $93(40.6)$ \\
\hline Spanish & $171(42.1)$ & $88(49.7)$ & $83(36.2)$ \\
\hline Both equally & $89(21.9)$ & $36(20.3)$ & $53(23.1)$ \\
\hline \multicolumn{4}{|l|}{ Personal language preference } \\
\hline English & $213(52.3)$ & $84(47.2)$ & $129(56.3)$ \\
\hline Spanish & $79(19.4)$ & $48(27.0)$ & $31(13.5)$ \\
\hline Both equally & $115(28.3)$ & $46(25.8)$ & $69(30.1)$ \\
\hline \multicolumn{4}{|c|}{ Mother's education (highest completed) } \\
\hline Less than primary school & $36(9.3)$ & $18(10.9)$ & $18(8.1)$ \\
\hline Primary/elementary school & $117(30.3)$ & $45(27.3)$ & $72(32.6)$ \\
\hline High school & $146(37.8)$ & $63(38.2)$ & $83(37.6)$ \\
\hline Any college or technical school & $87(22.5)$ & 39 (23.6) & $48(21.7)$ \\
\hline \multicolumn{4}{|l|}{ Residence in crowded conditions ${ }^{c}$} \\
\hline Yes & $130(31.9)$ & $69(38.8)$ & $61(26.6)$ \\
\hline No & $277(68.1)$ & $109(61.2)$ & $168(73.4)$ \\
\hline \multicolumn{4}{|c|}{ Participation in organized sports in the last 6 months } \\
\hline Yes & $162(39.8)$ & $89(50.0)$ & $73(31.9)$ \\
\hline No & $245(60.2)$ & $89(50.0)$ & $156(68.1)$ \\
\hline
\end{tabular}




\begin{tabular}{|c|c|c|c|}
\hline \multirow[t]{2}{*}{ Characteristic } & \multirow{2}{*}{$\frac{\text { Overall }(\mathrm{n}=410)^{a}}{\mathrm{~N}(\%)}$} & \multirow{2}{*}{$\frac{\text { Males }(\mathbf{n}=180)}{N(\%)}$} & \multirow{2}{*}{$\frac{\text { Females }(\mathbf{n}=230)}{N(\%)}$} \\
\hline & & & \\
\hline \multicolumn{4}{|l|}{ Sexually active ${ }^{d}$} \\
\hline Yes & $266(64.9)$ & $127(70.6)$ & $139(60.4)$ \\
\hline No & $144(35.1)$ & $53(29.4)$ & $91(39.6)$ \\
\hline \multicolumn{4}{|l|}{ Current gang membership or affiliation } \\
\hline Yes & $65(16.1)$ & $24(13.7)$ & $41(18.0)$ \\
\hline No & $338(83.9)$ & $151(86.3)$ & $187(82.0)$ \\
\hline \multicolumn{4}{|l|}{ Sexual partner in a gang } \\
\hline Yes & $54(13.2)$ & $18(10.0)$ & $36(15.7)$ \\
\hline No & $183(44.6)$ & $93(51.7)$ & $90(39.1)$ \\
\hline No partners reported & $173(42.2)$ & $69(38.3)$ & $104(45.2)$ \\
\hline \multicolumn{4}{|l|}{ Regular church attendance } \\
\hline Yes & $180(44.1)$ & $79(44.4)$ & $101(43.9)$ \\
\hline No & $228(55.8)$ & $99(55.6)$ & $129(56.1)$ \\
\hline \multicolumn{4}{|l|}{ Current student status } \\
\hline Yes, less than high school & $291(72.0)$ & $109(62.6)$ & $182(79.1)$ \\
\hline Yes, college or technical school (completed HS or GED) & $49(12.1)$ & $29(16.7)$ & $20(8.7)$ \\
\hline No, completed at least high school/GED & $21(5.2)$ & $12(6.9)$ & $9(3.9)$ \\
\hline No, not completed high school/GED & $43(10.6)$ & $24(13.8)$ & $19(8.3)$ \\
\hline \multicolumn{4}{|l|}{ Parental monitoring $e$} \\
\hline No adult caretaker & $55(13.5)$ & $29(16.2)$ & $26(11.3)$ \\
\hline High levels & $183(44.7)$ & $62(34.6)$ & $121(52.6)$ \\
\hline Less than high levels & $171(41.8)$ & $88(49.2)$ & $83(36.1)$ \\
\hline
\end{tabular}

GED $=$ General Education Development test.

${ }^{a}$ Numbers may not add to 410 due to missing data.

${ }^{b}$ Excludes one participant with no available data. Mean age $=16.4$ years, 16.1 (females), 16.8 (males); $p<.01$.

${ }^{c}$ According to the U.S. Census definition of more than one person per room in the household (including bedrooms, bathrooms, living rooms, etc.).

$d_{\text {Reporting vaginal and/or anal sex at baseline. }}$

${ }^{e}$ Parental monitoring was computed as the average score on a 4-question scale. Participants with average monitoring corresponding to "most to all of the time" were considered to have high levels of parental monitoring. 
Table 2

Attitudes and use of alcohol and marijuana at baseline and during follow-up among 410 Latino Youth, San Francisco, California, 2001-2004

\begin{tabular}{|c|c|c|c|}
\hline Characteristic & $\frac{\text { Overall }(\mathrm{n}=410)^{a}}{\mathrm{~N}(\%)}$ & $\frac{\text { Males }(\mathrm{n}=180)}{\mathrm{N}(\%)}$ & $\frac{\text { Females }(\mathrm{n}=\mathbf{2 3 0})}{\mathrm{N}(\%)}$ \\
\hline \multicolumn{4}{|l|}{ Attitudes $b$} \\
\hline \multicolumn{4}{|c|}{ Perceived peer attitudes toward alcohol use } \\
\hline Disapprove & $95(23.2)$ & $44(24.4)$ & $51(22.2)$ \\
\hline Don't care & $225(54.9)$ & $107(59.4)$ & $118(51.3)$ \\
\hline Approve & $90(22.0)$ & $29(16.1)$ & $61(26.5)$ \\
\hline \multicolumn{4}{|c|}{ Perceived parental attitudes toward alcohol use } \\
\hline Disapprove & $377(92.0)$ & $164(91.1)$ & $213(92.6)$ \\
\hline Don't care & $22(5.4)$ & $9(5.0)$ & $13(5.7)$ \\
\hline Approve & $11(2.7)$ & $7(3.9)$ & $4(1.7)$ \\
\hline \multicolumn{4}{|c|}{ Perceived peer attitudes toward marijuana use } \\
\hline Disapprove & $91(22.3)$ & $46(25.8)$ & $45(19.6)$ \\
\hline Don't care & $203(49.8)$ & $92(51.7)$ & $111(48.3)$ \\
\hline Approve & $114(27.9)$ & $40(22.5)$ & $74(32.2)$ \\
\hline \multicolumn{4}{|c|}{ Perceived parental attitudes toward marijuana use } \\
\hline Disapprove & $383(93.4)$ & $164(91.1)$ & $219(95.2)$ \\
\hline Don't care & $18(4.4)$ & $10(5.6)$ & $8(3.5)$ \\
\hline Approve & $9(2.2)$ & $6(3.3)$ & $3(1.3)$ \\
\hline \multicolumn{4}{|l|}{ Alcohol use } \\
\hline \multicolumn{4}{|c|}{ Alcohol use in last 6 months (enrollment) } \\
\hline Yes & $310(76.2)$ & $137(77.0)$ & $173(75.6)$ \\
\hline No & $97(23.8)$ & $41(23.0)$ & $56(24.4)$ \\
\hline \multicolumn{4}{|l|}{ Alcohol use during study } \\
\hline Reported any use at baseline & $310(76.7)$ & $137(77.0)$ & $173(76.6)$ \\
\hline First reported use during follow-up & $67(16.6)$ & $31(17.4)$ & $36(15.9)$ \\
\hline None reported & $27(6.7)$ & $10(5.6)$ & $17(7.5)$ \\
\hline \multicolumn{4}{|l|}{ Highest use frequency reported ${ }^{c}$} \\
\hline Daily & $31(8.2)$ & $20(11.9)$ & $11(5.3)$ \\
\hline At least once a week & $164(43.6)$ & $81(48.2)$ & $83(39.9)$ \\
\hline At least once a month & $75(19.9)$ & $32(19.1)$ & $43(20.7)$ \\
\hline Less than once a month & $61(16.2)$ & $19(11.3)$ & $42(20.2)$ \\
\hline A couple of times & $45(12.0)$ & $16(9.5)$ & $29(13.9)$ \\
\hline \multicolumn{4}{|c|}{ Highest frequency of getting drunk in last 6 months ${ }^{c}$} \\
\hline Daily or weekly & $119(31.6)$ & $70(41.7)$ & $49(23.6)$ \\
\hline At least once a month & $77(20.5)$ & $31(18.5)$ & $46(22.1)$ \\
\hline Less than once a month & $45(12.0)$ & $14(8.3)$ & $31(14.9)$ \\
\hline A couple of times & $77(20.5)$ & $29(17.3)$ & $48(23.1)$ \\
\hline
\end{tabular}




\begin{tabular}{|c|c|c|c|}
\hline \multirow[t]{2}{*}{ Characteristic } & \multirow{2}{*}{$\frac{\text { Overall }(\mathrm{n}=410)^{a}}{\mathrm{~N}(\%)}$} & \multirow{2}{*}{$\frac{\text { Males }(\mathbf{n}=180)}{N(\%)}$} & \multirow{2}{*}{$\frac{\text { Females }(\mathbf{n}=\mathbf{2 3 0})}{N(\%)}$} \\
\hline & & & \\
\hline Never & $58(15.4)$ & $24(14.3)$ & $34(16.4)$ \\
\hline \multicolumn{4}{|l|}{ Marijuana use } \\
\hline \multicolumn{4}{|l|}{ Where marijuana could be obtained $d$} \\
\hline From boy/girl friend & $122(29.8)$ & $37(20.6)$ & $85(37.0)$ \\
\hline From friends & $334(81.5)$ & $146(81.1)$ & $188(81.7)$ \\
\hline From regular supplier (not a friend) & $199(48.5)$ & $96(53.3)$ & $103(44.8)$ \\
\hline From a relative & $102(29.0)$ & $34(21.8)$ & $68(33.0)$ \\
\hline Grow it & $71(17.3)$ & $38(21.1)$ & $33(14.3)$ \\
\hline \multicolumn{4}{|l|}{ Highest amount of money spent on marijuana $e$} \\
\hline Nothing & $51(25.1)$ & $14(17.9)$ & $37(29.6)$ \\
\hline$\$ 1-20$ & $118(58.1)$ & $43(55.1)$ & $75(60.0)$ \\
\hline$\$ 21-100$ & $26(12.8)$ & $15(19.2)$ & $11(8.8)$ \\
\hline$>\$ 100$ & $8(3.9)$ & $6(7.7)$ & $2(1.6)$ \\
\hline \multicolumn{4}{|l|}{ Marijuana use in previous 6 months (baseline) } \\
\hline Yes & $227(55.4)$ & $94(52.2)$ & $133(57.8)$ \\
\hline No & $183(44.6)$ & $86(47.8)$ & $97(42.2)$ \\
\hline \multicolumn{4}{|l|}{ Marijuana use during study } \\
\hline Reported any use in last 6 months, baseline & $227(56.9)$ & $94(54.0)$ & $133(59.1)$ \\
\hline Re-/Initiated marijuana use in follow-up & $83(20.8)$ & $42(24.1)$ & $41(18.2)$ \\
\hline None reported & $89(22.3)$ & $38(21.8)$ & $51(22.7)$ \\
\hline \multicolumn{4}{|l|}{ Where marijuana is regularly used $f$} \\
\hline At home & $61(30.7)$ & $27(32.5)$ & $34(29.3)$ \\
\hline At a friend's home & $149(74.5)$ & $57(67.9)$ & $92(79.3)$ \\
\hline On the street or in parking lots & $155(77.5)$ & $65(77.4)$ & $90(77.6)$ \\
\hline At school & $45(22.5)$ & $22(26.2)$ & $23(19.8)$ \\
\hline At a park & $125(62.5)$ & $54(64.3)$ & $71(61.2)$ \\
\hline At a movie theater or mall & $41(20.5)$ & $24(28.6)$ & $17(14.7)$ \\
\hline In a car & $100(71.4)$ & $44(73.3)$ & $56(70.0)$ \\
\hline \multicolumn{4}{|l|}{ Highest use frequency reported $g$} \\
\hline Daily & $95(30.9)$ & $44(32.4)$ & $51(29.8)$ \\
\hline Weekly & $80(26.1)$ & $43(31.6)$ & 37 (21.6) \\
\hline At least once a month & $44(14.3)$ & $16(11.8)$ & $28(16.4)$ \\
\hline Less than once a month & $27(8.8)$ & $9(6.6)$ & $18(10.5)$ \\
\hline A couple of times & $61(19.9)$ & $24(17.7)$ & 37 (21.6) \\
\hline
\end{tabular}

\footnotetext{
${ }^{a}$ Numbers may not add to 410 due to missing follow-up data on some participants. Percents may not add to 100 due to rounding.

${ }^{b}$ As measured at study enrollment.

${ }^{c}$ Limited to the 377 participants who reported any alcohol use during the study. Frequency was assessed for each 6 month period. Numbers may not add to 377 due to missing data.

${ }^{d}$ Options are not mutually exclusive; number indicates the number of participants who indicated source.
} 
${ }^{e}$ Highest amount spent at a single time. Limited to the 227 participants who reported any marijuana use in the previous 6 months. Missing for $11 \%$ of the 227. Mean amount reported $=\$ 21.60$.

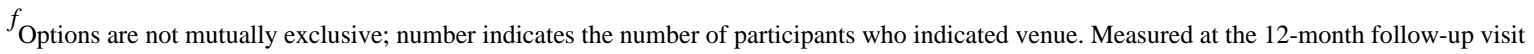
among participants who reported use in the previous 6 months. Marijuana use in a car was measured at the final (30 month) follow-up visit.

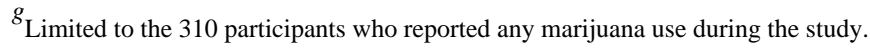


Table 3

Baseline predictors of alcohol trajectory group affiliation with multivariable multinomial logistic regression among Latino youth in San Francisco, California, 2001-2004 ${ }^{a}$

\begin{tabular}{|c|c|c|c|c|}
\hline \multirow[t]{2}{*}{ Characteristic } & \multicolumn{2}{|c|}{ Group 1 Low users } & \multicolumn{2}{|c|}{ Group 3 Frequent users } \\
\hline & OR & $95 \% \mathrm{CI}$ & OR & $95 \% \mathrm{CI}$ \\
\hline \multicolumn{5}{|l|}{ Gender* } \\
\hline Male & 1.27 & $(.58,2.76)$ & 4.41 & $(2.12,9.19)$ \\
\hline Female & Referent & - & Referent & - \\
\hline Age (years) ${ }^{*}$ & .60 & $(.45, .82)$ & .92 & $(.72,1.22)$ \\
\hline \multicolumn{5}{|c|}{ Personal language preference ${ }^{*}$} \\
\hline English & Referent & - & Referent & - \\
\hline Spanish & 6.23 & $(2.08,18.68)$ & .46 & $(.16,1.31)$ \\
\hline Both equally & 1.14 & $(.46,2.82)$ & .51 & $(.23,1.14)$ \\
\hline \multicolumn{5}{|l|}{ Sexually active ${ }^{*}$} \\
\hline Yes & 1.29 & $(.60,2.76)$ & 4.26 & $(1.97,9.23)$ \\
\hline No & Referent & - & Referent & - \\
\hline \multicolumn{5}{|l|}{ Any gang exposure ${ }^{*}, b$} \\
\hline Yes & .72 & $(.24,2.14)$ & 2.46 & $(1.08,5.60)$ \\
\hline No & Referent & - & Referent & - \\
\hline \multicolumn{5}{|c|}{ Regular church attendance } \\
\hline Yes & .45 & $(.21, .98)$ & .55 & $(.28,1.10)$ \\
\hline No & Referent & - & Referent & - \\
\hline \multicolumn{5}{|l|}{ Parental monitoring $*$} \\
\hline No adult caretaker & .34 & $(.07,1.69)$ & 3.36 & $(1.18,9.55)$ \\
\hline High levels & Referent & - & Referent & - \\
\hline Less than high levels & .34 & $(.14, .78)$ & 2.17 & $(1.05,4.46)$ \\
\hline \multicolumn{5}{|c|}{ Perceived peer attitudes toward alcohol use } \\
\hline Disapprove & Referent & - & Referent & - \\
\hline Don't care & .89 & $(.39,2.02)$ & 2.63 & $(.96,7.17)$ \\
\hline Approve & .26 & $(.05,1.28)$ & 2.78 & $(.91,8.45)$ \\
\hline \multicolumn{5}{|c|}{$* x<.05$ for Wald Test of all $\beta=0$. } \\
\hline${ }^{a}$ Reference group is Gr & & & & \\
\hline
\end{tabular}


Table 4

Baseline predictors of marijuana trajectory group affiliation with multivariable multinomial logistic regression among Latino youth in San Francisco, California, 2001-2004

\begin{tabular}{|c|c|c|c|c|}
\hline \multirow[t]{2}{*}{ Characteristic } & \multicolumn{2}{|c|}{ Group 1 Low users } & \multicolumn{2}{|c|}{ Group 3 Frequent users } \\
\hline & OR & $95 \% \mathrm{CI}$ & OR & $95 \% \mathrm{CI}$ \\
\hline \multicolumn{5}{|l|}{ Gender } \\
\hline Male & .75 & $(.38,1.45)$ & 1.57 & $(.77,3.17)$ \\
\hline Female & Referent & - & Referent & \\
\hline Age (years) & .90 & $(.73,1.12)$ & 1.00 & $(.79,1.27)$ \\
\hline \multicolumn{5}{|c|}{ Personal language preference ${ }^{*}$} \\
\hline English & Referent & - & Referent & - \\
\hline Spanish & 3.53 & $(1.48,8.45)$ & .08 & $(.01, .51)$ \\
\hline Both equally & 1.97 & $(.96,4.04)$ & .41 & $(.19, .88)$ \\
\hline \multicolumn{5}{|l|}{ Sexually active ${ }^{*}$} \\
\hline Yes & .47 & $(.24, .95)$ & 2.13 & $(.93,4.89)$ \\
\hline No & Referent & - & Referent & - \\
\hline \multicolumn{5}{|l|}{ Any gang exposure ${ }^{*}, b$} \\
\hline Yes & .30 & $(.13, .71)$ & 1.50 & $(.73,3.09)$ \\
\hline No & Referent & - & Referent & \\
\hline \multicolumn{5}{|c|}{ Regular church attendance } \\
\hline Yes & 1.02 & $(.55,1.90)$ & .95 & $(.48,1.88)$ \\
\hline No & Referent & - & Referent & \\
\hline \multicolumn{5}{|c|}{ Participation in organized sports in last 6 months } \\
\hline Yes & .86 & $(.46,1.60)$ & .52 & $(.26,1.06)$ \\
\hline No & Referent & & Referent & \\
\hline \multicolumn{5}{|l|}{ Parental monitoring ${ }^{*}$} \\
\hline No adult caretaker & .89 & $(.30,2.62)$ & 1.57 & $(.55,4.49)$ \\
\hline High levels & Referent & - & Referent & - \\
\hline Less than high levels & .49 & $(.25, .97)$ & 1.74 & $(.83,3.61)$ \\
\hline \multicolumn{5}{|c|}{ Perceived peer attitudes toward marijuana use ${ }^{*}$} \\
\hline Disapprove & Referent & - & Referent & - \\
\hline Don't care & .40 & $(.19, .87)$ & 1.63 & $(.46,5.73)$ \\
\hline Approve & .68 & $(.25,1.88)$ & 3.28 & $(.84,12.80)$ \\
\hline \multicolumn{5}{|c|}{$*^{*}<.05$ for Wald Test of all $\beta=0}$. \\
\hline${ }^{a}$ Reference group is Gro & & & & \\
\hline
\end{tabular}

